\title{
Genetic counselling in a national referral centre for pulmonary hypertension
}

\author{
Barbara Girerd ${ }^{1,2,3,6}$, David Montani ${ }^{1,2,3,6}$, Xavier Jaïs ${ }^{1,2,3}$, Mélanie Eyries ${ }^{4}$, \\ Azzedine Yaici ${ }^{1,2,3}$, Benjamin Sztrymf ${ }^{1,2,3}$, Laurent Savale ${ }^{1,2,3}$, \\ Florence Parent ${ }^{1,2,3}$, Florence Coulet ${ }^{4}$, Laurent Godinas ${ }^{1,2,3}$, \\ Edmund M. Lau ${ }^{1,2,5^{\prime}}$, Yuichi Tamura ${ }^{1,2,3}$, Olivier Sitbon ${ }^{1,2,3}$, Florent Soubrier ${ }^{4}$, \\ Gérald Simonneau ${ }^{1,2,3}$ and Marc Humbert ${ }^{1,2,3}$
}

Affiliations: ${ }^{1}$ Université Paris Sud, Faculté de Médecine, Université Paris-Saclay, Le Kremlin Bicêtre, France. ${ }^{2} \mathrm{AP}-\mathrm{HP}$, Centre de Référence de l'Hypertension Pulmonaire Sévère, Département Hospitalo-Universitaire (DHU) Thorax Innovation (TORINO), Service de Pneumologie, Hôpital de Bicêtre, Le Kremlin Bicêtre, France. ${ }^{3}$ UMR S999, Université Paris-Sud, INSERM, Laboratoire d'Excellence (LabEx) en Recherche sur le Médicament et l'Innovation Thérapeutique (LERMIT), Centre Chirurgical Marie Lannelongue, Le Plessis Robinson, France. 'UMR_S956, Université Pierre et Marie Curie-Paris 6, INSERM, Laboratoire d'Oncogénétique et Angiogénétique Moléculaire, Groupe Hospitalier Pitié-Salpétrière, Paris, France. ${ }^{5}$ Sydney Medical School, University of Sydney, Camperdown, Australia. ${ }^{6}$ Both authors contributed equally to this work.

Correspondence: Marc Humbert, Université Paris-Sud, Service de Pneumologie, Hôpital Bicêtre, 78 Rue du général Leclerc, 94270 Le Kremlin Bicêtre, France. E-mail: marc.humbertabct.aphp.fr

ABSTRACT Genetic causes of pulmonary arterial hypertension (PAH) and pulmonary veno-occlusive disease (PVOD) have been identified, leading to a growing need for genetic counselling.

Between 2003 and 2014, genetic counselling was offered to 529 PAH and 100 PVOD patients at the French Referral Centre for Pulmonary Hypertension.

Mutations in PAH-predisposing genes were identified in 72 patients presenting as sporadic PAH (17\% of cases; 62 mutations in BMPR2, nine in ACVRL1 (ALK1) and one in ENG) and in 94 patients with a PAH family history ( $89 \%$ of cases; 89 mutations in BMPR2, three in ACVRL1 (ALK1) and two in KCNK3). Bi-allelic mutations in EIF2AK4 were identified in all patients with a family history of PVOD $(\mathrm{n}=19)$ and in seven patients $(8.6 \%)$ presenting as sporadic PVOD. Pre-symptomatic genetic diagnosis was offered to 272 relatives of heritable $\mathrm{PAH}$ patients, identifying mutations in $36.4 \%$ of them. A screening programme is now offered to asymptomatic mutation carriers to detect PAH in an early phase and to identify predictors of outcomes in asymptomatic BMPR2 mutation carriers. BMPR2 screening allowed us to offer pre-implantation diagnosis to two couples with a BMPR2 mutation.

Genetic counselling can be implemented in pulmonary hypertension centres.

@ERSpublications

Genetic causes of PAH and PVOD have been identified; genetic counselling can be implemented in $\mathrm{PH}$ centres http://ow.ly/TLbee

For editorial comment see Eur Respir J 2016; 47: 388-389 [DOI: 10.1183/13993003.01858-2015].

This article has supplementary material available from erj.ersjournals.com

Received: May 072015 | Accepted after revision: Oct 142015 | First published online: Dec 232015

Conflict of interest: Disclosures can be found alongside the online version of this article at erj.ersjournals.com

Support statement: L. Godinas is supported by the European Respiratory Society (grant LTRF-2013-1592). Y. Tamura (RESPIRE2-2013-4919) is the recipient of an ERS/EU RESPIRE2 Marie Curie research fellowship.

Copyright @ERS 2016 


\section{Introduction}

Pulmonary arterial hypertension (PAH) is a rare and severe disease affecting small pulmonary arteries, with abnormal proliferation of pulmonary artery smooth muscle cells and endothelial cells leading to an increase in pulmonary vascular resistance and right ventricular failure [1-3]. PAH may occur in a number of different clinical contexts such as connective tissue diseases, HIV infection, congenital heart diseases, portal hypertension and anorexigen exposure [4]. When sporadic disease occurs without any family history or predisposing conditions, PAH is referred to as idiopathic. In 1954, DRESDALE et al. [5] described the first cases of PAH occurring in a familial context, suggesting the existence of a heritable form of the disease. Since then, many familial PAH cases have been described, allowing for an understanding that heritable PAH is transmitted as an autosomal dominant trait with incomplete penetrance. Although genetic anticipation was previously suggested to occur in heritable $\mathrm{PAH}$, this was recently demonstrated to be an artefact related to incomplete time of observation of kindred carriers of mutations in PAH-predisposing genes [6].

In 2000, mutations of the BMPR2 (bone morphogenetic protein receptor type II) gene were identified as the major causes of heritable PAH. This gene encodes a type II receptor (BMPRII) belonging to the superfamily of transforming growth factor (TGF)- $\beta$ [7-10]. The BMPRII receptor was originally described to be involved in the regulation of growth and differentiation of bone and cartilage [11, 12], but more recently, it was also shown to play a critical role in the regulation of growth, differentiation and apoptosis of other cell types, including endothelial cells and pulmonary artery smooth muscle cells [13].When PAH occurs in a familial context, germline mutations in BMPR2 are detected in at least 70\% of cases [14-19]. BMPR2 mutations can also be detected in $3.5-40 \%$ of apparently sporadic PAH cases [14-16, 20]. The occurrence of $\mathrm{PAH}$ in patients with a personal or family history of hereditary haemorrhagic telangiectasia (HHT) has allowed two other genes involved in the development of both PAH and HHT to be identified: ACVRL1 (ALK1) (activin A receptor type II-like kinase 1) and ENG (endoglin) [16, 21]). Rare mutations in genes coding for Smad proteins involved in the TGF- $\beta$ signalling pathway have also been reported (SMAD9 [22, 23], and SMAD1 and SMAD4 [22]). In 2012, Austin et al. [24] discovered the involvement of $C A V-1$ (caveolin-1) mutations in $\mathrm{PAH}$, using whole-exome sequencing to study a third-generation multiple-affected family members with PAH. In 2013, MA et al. [25] demonstrated the involvement of a channelopathy of KCNK3 (potassium channel, two pore domain subfamily K, member 3 ) in the development of heritable PAH, identifying three familial PAH patients and three idiopathic PAH patients carrying a mutation in the KCNK3 gene.

Pulmonary veno-occlusive disease (PVOD) is a rare disorder that can be misdiagnosed as PAH [26], but pulmonary vascular remodelling predominates in the pulmonary venules and small veins in PVOD [27, 28]. Recently, we have demonstrated the involvement of bi-allelic mutations in the EIF2AK4 (eukaryotic translation initiation factor $2 \alpha$ kinase 4 ) gene in the development of heritable PVOD [19, 29]. PVOD due to EIF2AK4 mutations is an autosomal recessive disease with an unknown penetrance. Rare PVOD cases have been reported in patients carrying BMPR2 mutations [27, 28], but a careful clinical and pathological de novo evaluation of these cases should be undertaken, together with a search for EIF2AK4 mutations.

Since January 2003, the French Referral Centre for Pulmonary Hypertension has offered genetic counselling and testing to all patients with PAH considered to be sporadic, familial or induced by anorexigen exposure, and in sporadic and familial PVOD patients. Here, we review our 12-year experience in genetic counselling for pulmonary hypertension.

\section{Materials and methods}

Patients

$\mathrm{PAH}$ was defined as a mean pulmonary artery pressure (mPAP) $\geqslant 25 \mathrm{mmHg}$ with a normal pulmonary artery wedge pressure $(\leqslant 15 \mathrm{mmHg})$ measured during right heart catheterisation after other causes of pre-capillary pulmonary hypertension had been eliminated. Haemodynamic evaluation by right heart catheterisation was performed at baseline in all subjects according to our previously described protocol $[30,31]$. Idiopathic PAH was diagnosed after exclusion of associated conditions and absence of a family history of PAH, as summarised in the updated classification system [4]. Familial PAH was recognised if there was more than one confirmed cases of PAH in the family.

PVOD was considered as confirmed in the presence of extensive and diffuse obstruction of pulmonary veins and venules on lung biopsies, post-mortem lung samples or lungs obtained after lung transplantation. PVOD was considered "highly probable" when patients fulfilled the following characteristics: pre-capillary pulmonary hypertension, presence of two or more radiological abnormalities characteristic of PVOD on high-resolution computed tomography of the chest (lymph node enlargement, centrilobular ground-glass opacities and interlobular septal lines) and low diffusing capacity of the lung for carbon monoxide corrected for haemoglobin levels (DLCOc). The diagnosis of PVOD was also supported by the development of pulmonary oedema after initiation of specific PAH therapy [27, 31, 32]. 
French recommendations for genetic counselling

Genetic counselling follows the laws on bioethics (Decree 2008-321 of April 4, 2008) which set out the conditions for prescribing and conducting reviews of the genetic characteristics of a person and their identification by genetic fingerprinting for medical purposes and amending the Public Health Code (table 1).

The ethical principles of the French guidelines for genetic counselling and the 2015 conjoint European Respiratory Society (ERS) and European Society of Cardiology (ESC) guidelines for the diagnosis and treatment of pulmonary hypertension $[1,2]$ are to inform the patient properly to avoid harm, to allow the patient to preserve their autonomy (disclosure about the process, risks and benefits of the genetic testing without external pressures), and to allow equal access to genetic counselling and testing. We encourage patients to share genetic information with their family. A consultation with the psychologist is systematically offered to all patients and relatives, because of the psychological impact of genetic results.

To date, predictive genetic testing for children under the age of 18 years remains a matter of debate because of the absence of preventive and curative treatments in PAH. Thereby, in France, no genetic testing for PAH is currently offered to asymptomatic children under 18 years of age.

\section{Genetic testing at the French Referral Centre for Pulmonary Hypertension}

Since January 2003, the French Referral Centre for Pulmonary Hypertension has been offering genetic counselling and BMPR2 mutation screening (point mutations and large rearrangements) to all patients with $\mathrm{PAH}$ considered to be idiopathic, patients with a family history of $\mathrm{PAH}$, patients exposed to anorexigen drugs and patients with pulmonary hypertension associated with other rare diseases. When no mutation in BMPR2 was identified in patients with a family history of PAH, screening of ACVRL1 (ALK1) and ENG was performed (point mutations and large rearrangements). As ACVRL1 (ALK1) and ENG mutations are associated with complete penetrance for HHT only at an advanced age, clinical signs of HHT were sought in all patients seen at genetic counselling and screening for $A C V R L 1$ ( $A L K 1)$ mutations was performed in patients under 35 years of age or if clinical signs of HHT were present (recurrent epistaxes, mucocutaneous telangiectases and arteriovenous malformations, particularly in the pulmonary, hepatic, and cerebral circulation). If no mutations in BMPR2, ACVRL1 (ALK1) and ENG were identified in patients with a family history of PAH, screening of $B M P R 2$ promoter, $S M A D 9, K C N K 3$ and $C A V-1$ genes was subsequently performed.

We recently demonstrated the involvement of bi-allelic EIF2AK4 mutations as the major cause of heritable PVOD [29]. Since 2014, patients followed in our centre (prevalent or incident) with a confirmed or a "highly probable" PVOD were also tested for EIF2AK4 mutations. If no EIF2AK4 mutations were identified in a patient displaying "highly probable" PVOD or a family history of PAH/PVOD, screening for PAH-predisposing genes was performed. All patients provided written informed consent prior to undertaking genetic testing. These procedures follow the recent 2015 ECS/ERS guidelines [1, 2].

\section{TABLE 1 French guidelines for genetic counselling: key points of Decree 2008-321 of April 4, 2008}

The person is informed about the disease characteristics, detection techniques, genetic analysis sensitivity, prevention and treatment possibilities, genetic modality of the disease transmission, and consequences for the other family members.

Informed written consent is mandatory.

Patient under 18 years old and adults under guardianship must agree with the genetic testing, whereas the informed written consent must be signed by the legal representative.

The genetic counselling must be done in an individual consultation.

For asymptomatic high-risk relatives, the genetic counselling must be done in an individual consultation. This pre-symptomatic diagnosis requires a multidisciplinary approach involving geneticists, pulmonologists, genetic counsellors, psychologists and nurses. This team is registered to the biomedical Agency and follows their recommendations.

Genetic testing can be prescribed in asymptomatic high-risk relatives under 18 years old or in adults under guardianship, only if their family can benefit from preventive or curative treatments.

Genetic testing are performed under the responsibility of a physician allowed by Article R.1131-9, practicing in one of the institutions listed in Article R.1131-13. This physician is the only one authorised to sign the genetic results.

The genetic testing can only be performed in biological laboratories of public health institutions, cancer centres, biological laboratories mentioned in Article L.6211-2 and in biological laboratories of the French blood bank centres authorised by Article R.11301-14.

The prescriber informs directly the person who underwent the genetic testing in an individual consultation.

The person who underwent the genetic testing can refuse to know the result. 
Molecular methods

Screening for point mutations in the coding regions and intronic junctions of BMPR2, ACVRL1 (ALK1), ENG, SMAD9, KCNK3, CAV-1 and EIF2AK4

Human genomic DNA was prepared from whole-blood samples using a semi-automatised Extragene extractor (Genomic Industry, Archamps, France) and a DNA extraction kit (Promega France, Charbonnières-les-Bains, France) according to the manufacturer's standard protocol or DNA was extracted by column extraction with a QIAmp DNA blood kit (Qiagen, Courtaboeuf, France). All coding exons and intronic junctions of BMPR2, ACVRL1 (ALK1), ENG, SMAD9, KCNK3, EIF2AK4 and CAV-1 were amplified by PCR with intronic flanking primers and additional exonic primers when necessary. The 2066-bp sequences upstream of the initiation codon of BMPR2 were divided into five fragments for amplification of the $B M P R 2$ promoter (all PCR primer details are available upon request).

PCR-amplified products were purified and directly sequenced as previously described [16]. The resulting sequence data were analysed with SeqScape version 2.5 (Applied Biosystems, Foster City, CA, USA) in comparison with the GenBank reference sequences of BMPR2 (NM_001204.6), ACVRL1 (ALK1) (NM_000020.2), ENG (NM_001114753.1), SMAD9 (NM_005905.5), KCNK3 (NM_002246), EIF2AK4 (NM_001013703) and CAV-1 (NM_001753). In all cases, any sequence variation found was determined twice to confirm the observed changes. Nucleotide numbering follows cDNA numbering with +1 corresponding to the A of the ATG translation initiation codon in the reference sequence (www.hgvs.org/mutnomen).

Screening of large rearrangements of BMPR2, ACVRL1 (ALK1) and ENG

The SALSA multiplex ligation-dependent probe amplification P093 HHT probemix kit (MRC-Holland, Amsterdam, The Netherlands) was used to screen for rearrangements of one or more exons of BMPR2, ACVRL1 (ALK1) and ENG, according to the manufacturer's standard protocol. Fragment analysis of multiplex PCR was carried out on an ABI 3730 DNA analyser and results were analysed using GeneMapper version 4.0 (Applied Biosystems, Foster City, CA, USA). Two DNA samples from unaffected individual were used as controls in each experiment. Electrophoregrams were superimposed on those generated from control DNA by adjusting the peaks to the same level as obtained for the control amplicons.

\section{Results}

\section{Genetic counselling and testing in PAH patients}

Up to December 2014, genetic counselling and testing were offered to 529 PAH patients, including 106 patients from 77 families with a family history of PAH. In this cohort, 492 (93\%) patients were more than 18 years old at PAH. Mutations in PAH-predisposing genes were identified in $17 \%(n=72)$ of patients who were considered to have idiopathic or anorexigen-induced disease. In this population, we identified $62(86 \%)$ BMPR2 mutation carriers, nine (13\%) ACVRL1 (ALK1) mutation carriers and one (1\%) ENG mutation carrier (figure 1a). Mutations in PAH-predisposing genes were identified in $89 \%(\mathrm{n}=94)$ of familial PAH cases. Among them, 89 carried a BMPR2 mutation, three carried an ACVRL1 (ALK1) mutation and two carried a KCNK3 mutation (figure 1b). The distribution of mutations in PAH is presented in figure 2 and all mutations identified are listed in online supplementary table S1.

Of the 641 instances of proposed genetic counselling and testing, three patients refused the genetic consultation and nine declined the genetic testing after genetic counselling, which accounted for $<2 \%$ of patients. The reasons cited by patients included the inability to cope with a positive result or because of compliance to religious beliefs against genetic approaches.

\section{BMPR2 mutations identified in PAH patients}

We identified 127 different BMPR2 mutations (online supplementary table S1). These mutations were spread throughout BMPR2 with the exception of exon 13 (figure 3). We identified 29 missense mutations, 59 nonsense mutations, 13 splice defects, 23 deletions and one duplication, and two variants in the promoter region.

Although the cytoplasmic tail domain represents $50 \%$ of the size of the BMPRII protein, 87 out of the 101 point mutations $(86 \%)$ were localised in the kinase domain or in the extracellular domain of the BMPRII protein and only 14 point mutations (14\%) were localised in the cytoplasmic tail domain [33].

In order to estimate the frequency of de novo mutations in BMPR2, we selected a subgroup of PAH patients who were carriers of a BMPR2 mutation but without a family history of PAH. In these patients, we searched for the presence of the familial BMPR2 mutation in DNA samples from their parents. DNA samples were available from both parents in $13 \mathrm{PAH}$ patients fulfilling the above criteria. In four patients, the familial BMPR2 mutation was not detected in either parents and the possibility of incorrect paternity was excluded by the analysis of informative markers (figure 4). Three of these four patients (ABH058, 
a)

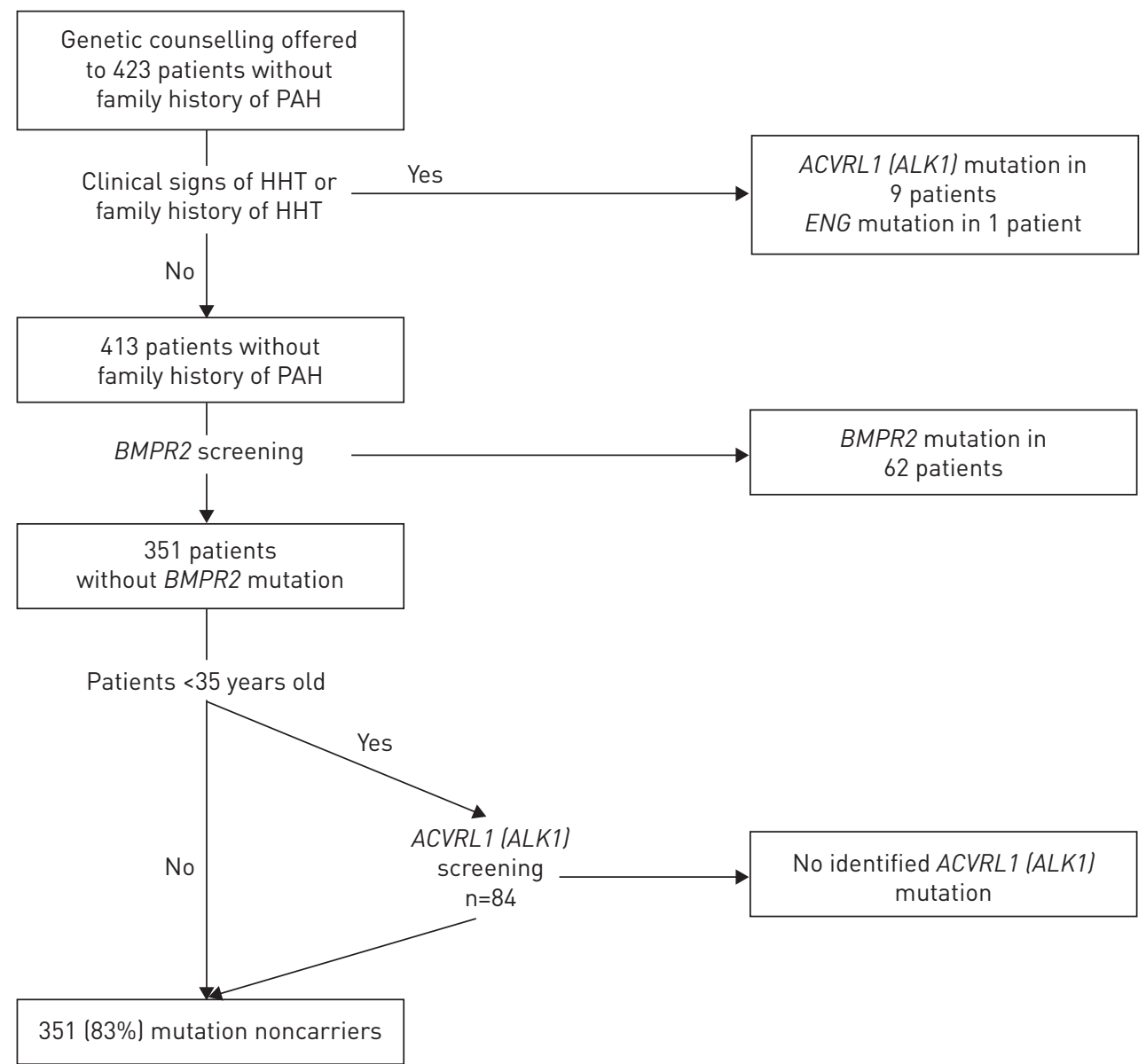

b)

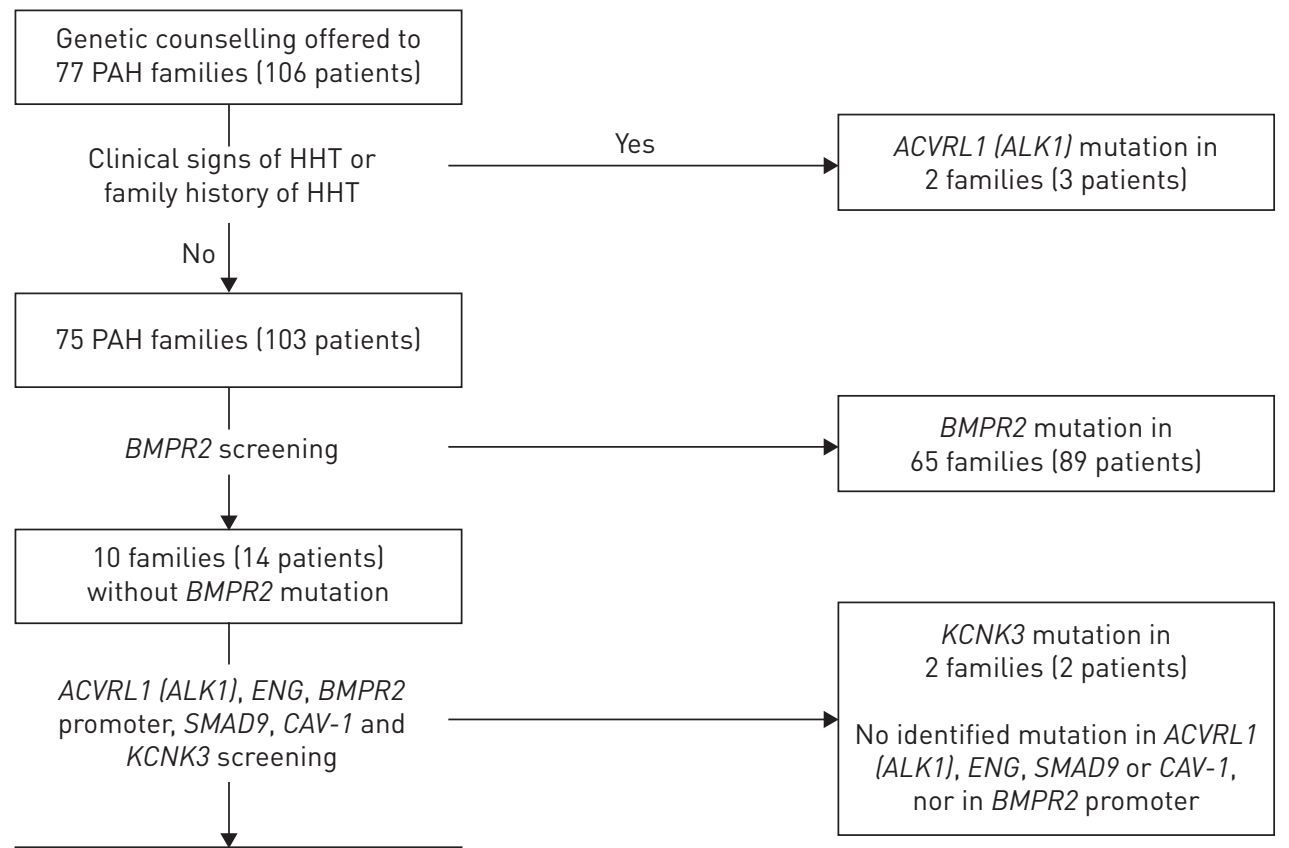

8 families $(10.4 \%, 12$ patients) without identified mutation

FIGURE 1 a) Screening of pulmonary arterial hypertension (PAH)-predisposing genes in PAH patients without a family history of PAH. b) Screening of PAH-predisposing genes in PAH families. HHT: hereditary haemorrhagic telangiectasia. 


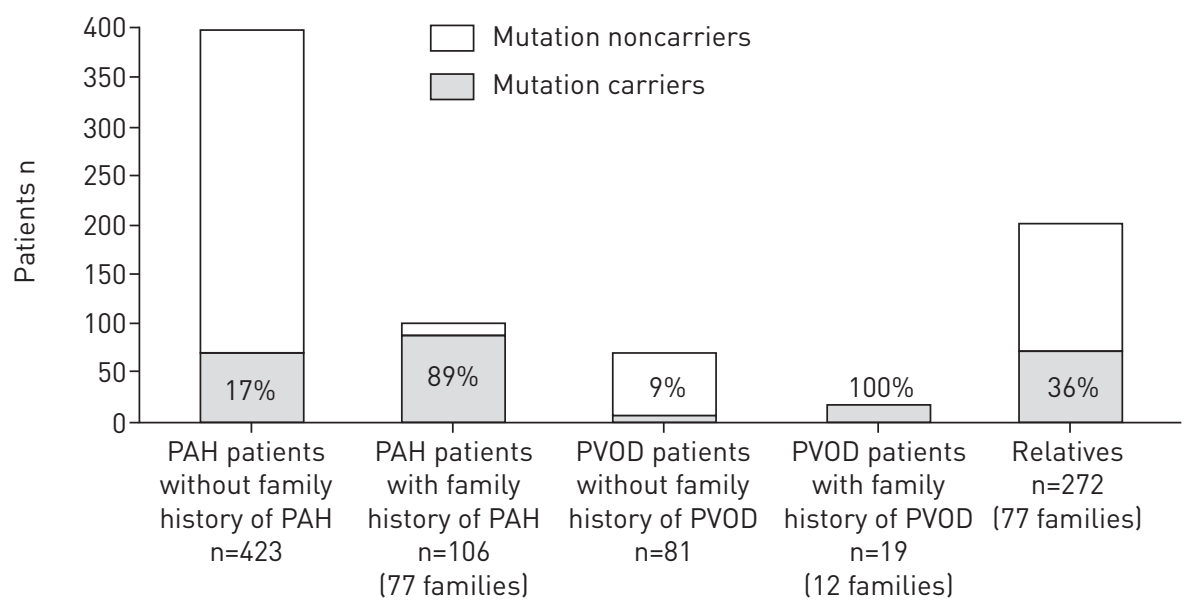

$\begin{array}{lccccc}\text { BMPR2 mutation } & 62 & 89 \text { (65 families) } & 0 & 0 & 96 \\ \text { ACVRL1 (ALK1) mutation } & 9 & 3 \text { (2 families) } & - & 0 & 1 \\ \text { ENG mutation } & 1 & 0 & - & 0 & - \\ \text { KCNK3 mutation } & - & 2 \text { (2 families) } & - & - & 2 \\ \text { EIF2AK4 mutations } & - & 0 & 74 & 7 & 19 \text { (12 families) } \\ \text { Total mutations } & 72 & 94 & 19\end{array}$

FIGURE 2 Distribution of mutations in sporadic and familial pulmonary arterial hypertension (PAH) and pulmonary veno-occlusive disease (PVOD) patients and asymptomatic relatives. -: untested.

$\mathrm{ABH} 100$ and $\mathrm{ABH} 177$ ) were carriers of a mutation previously identified in our cohort (figure 3 and online supplementary table S2). Mutation c.449dup identified in patient ABH086 was a unique mutation not identified in any other patients in our cohort. Clinical and haemodynamic characteristics at baseline of these four patients are presented in online supplementary table S2 and were similar to other heritable PAH cases $[16,34]$.

Ten PAH patients who had a family history of PAH were screened for mutation in the promoter region of BMPR2. Two variants of unknown significance were identified (online supplementary table S1). These variants are not described in human genetic variation databases (1000 Genomes or Exome Variant Server) and none of the $>400$ control alleles sequenced showed these variants. Using the bioinformatic prediction tool MatInspector, we identified a putative transcription factor binding site affected by one of these variants (c.-1585G>A). However functional analysis would be necessary to characterise the effect of these variants on the transcriptional activity of $B M P R 2$ before we can conclude on their potential deleterious effect.

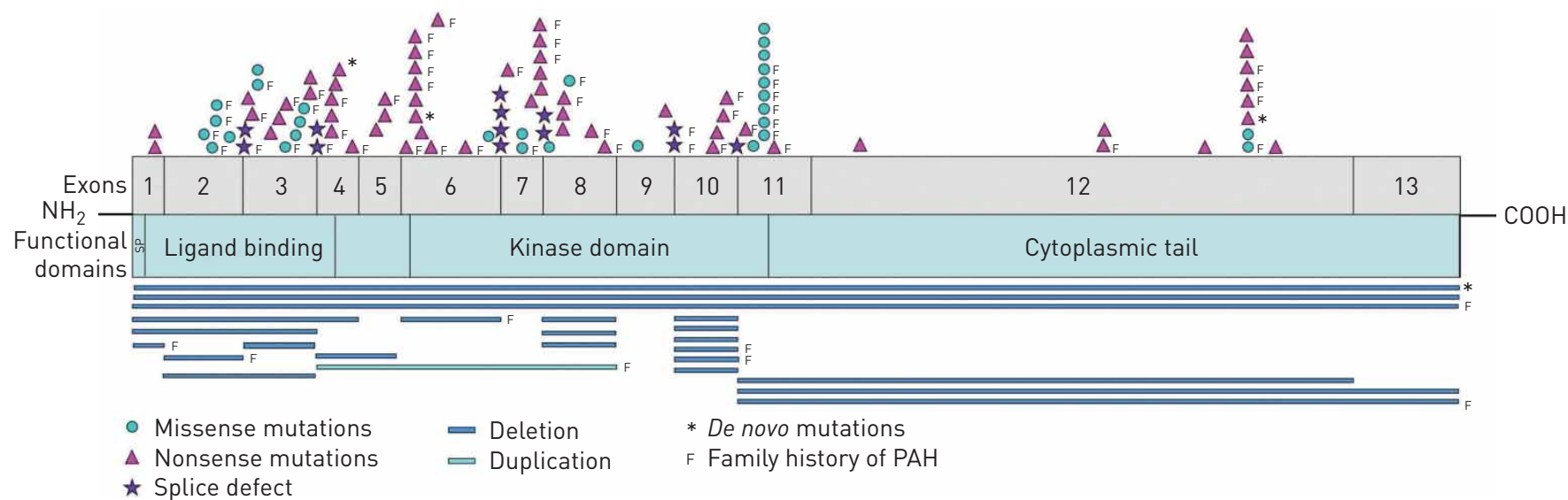

FIGURE 3 Localisation of BMPR2 mutations identified in pulmonary arterial hypertension (PAH) patients followed at the French Referral Centre for Pulmonary Hypertension. SP: signal peptide. 
Family ABH058

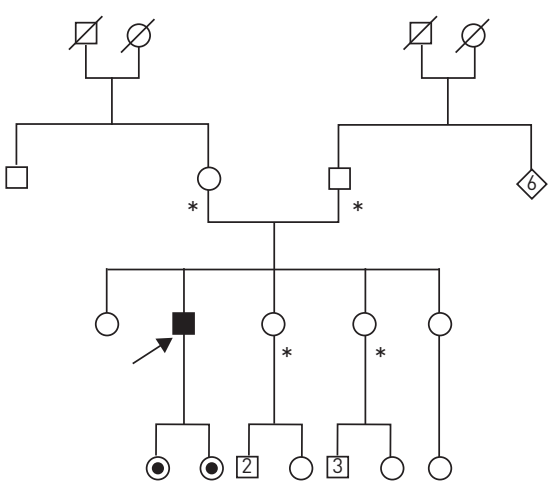

Family $\mathrm{ABH} 100$

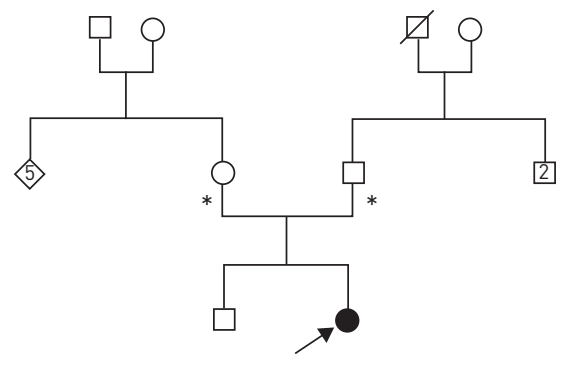

Family ABH086

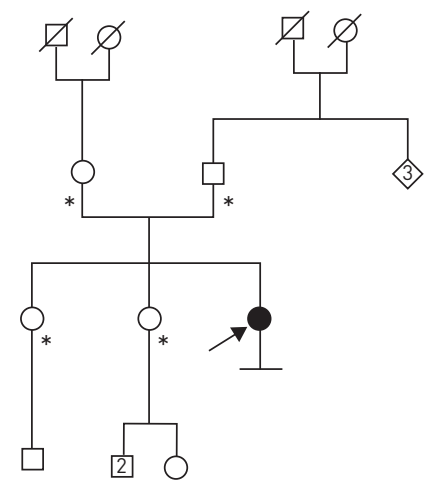

Family ABH177

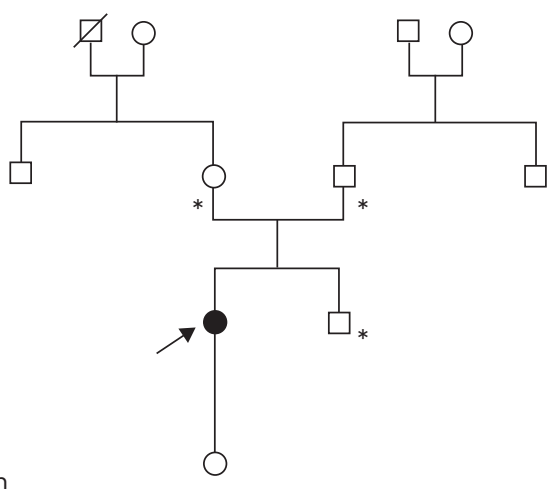

- Asymptomatic carriers of the familial BMPR2 mutation

* Noncarriers of the familial BMPR2 mutation

FIGURE 4 Family trees of patients displaying de novo mutation of BMPR2.

\section{ACVRL1 (ALK1) and ENG mutations identified in PAH patients}

At diagnosis, 10 patients displaying a sporadic form of $\mathrm{PAH}$ and three patients (from two different families) displaying a familial form of PAH presented with sign or family history of HHT. ACVRL1 (ALK1) and $E N G$ were screened in the first instance in such cases. A mutation in ACVRL1 (ALK1) was identified in nine of the 10 sporadic patients and in both familial forms of $\mathrm{PAH}$ (figures 1 and 2). As previously reported [16], the mutations in ACVRL1 (ALK1) are frequently localised in exon 10 and more particularly in the region encoding for the NANDOR box of the ALK1 protein. The remaining sporadic patient carried an ENG mutation (online supplementary table S1) [21]. This mutation was previously described in HHT patients (mutation reported in the HHT Mutation Database: http://www.hhtmutation.org).

Moreover, 84 sporadic PAH patients under 35 years old and 10 families in whom no mutations in the $B M P R 2$ gene were identified were screened for mutations in ACVRL1 (ALK1). No mutation was identified. However, three variants of unknown significance of ACVRL1 (ALK1) were identified: c.1049-5C $>$ G; c.293A >G; c.314-16T>C. The variant c.293A >G was previously described in HHT patients (variant described in the HHT Mutation Database: www.hhtmutation.org).

\section{SMAD9, CAV-1 and KCNK3 mutation screening in PAH patients}

Ten unrelated patients with familial PAH in whom no mutations in BMPR2, ACVRL1 (ALK1) and ENG were identified were screened for mutations in $S M A D 9, C A V-1$ and $K C N K 3$. No mutations were identified in SMAD9 and CAV-1 (figures 1 and 2). However, mutations in KCNK3 were identified in two unrelated patients (figures $1 \mathrm{~b}$ and 2) [25].

As scientific research, in order to estimate the frequency of KCNK3 mutations in the sporadic PAH population, 14 additional sporadic PAH patients were sequenced for $K C N K 3$ mutations and no mutations were identified [25]. 
EIF2AK4 mutation screening in PAH patients

The eight remaining heritable PAH families, in whom no mutations in BMPR2, ACVRL1 (ALK1), ENG, SMAD9, CAV-1 and KCNK3 were identified, were screened for mutations in EIF2AK4. No mutations were identified (figure 2).

\section{Genetic counselling and testing in PVOD patients}

Genetic counselling and testing were offered to 100 PVOD patients, 96 of them were more than 18 years old at PAH diagnosis We identified 19 patients from 12 families displaying a familial form of PVOD. In all families, we identified bi-allelic mutations in EIF2AK4 (figure 2 and online supplementary table S2). We also tested 81 patients displaying sporadic PVOD. Bi-allelic EIF2AK4 mutations were identified in seven sporadic PVOD patients (9\%).

17 EIF2AK4 mutation carriers were previously described [29] and we reported novel mutations identified in two heritable PVOD patients (c.2609C $>$ T, p.Ala870Val and c.4910C $>$ T, p.Leu1637Pro mutations; c.3633dup, p.His1212 $\mathrm{Thrfs}^{\star} 8$ and $4318 \mathrm{C}>\mathrm{T}$, p.Gln1440* mutations).

All sporadic PVOD cases without EIF2AK4 mutation were screened for BMPR2 mutations and no mutations were identified. Before the recent discovery of the involvement of EIF2AK4 mutations in PVOD, all familial PVOD cases were screened for BMPR2 and ACVRL1 (ALK1) mutations, and five PVOD families were screened for KCNK3, CAV-1 and SMAD9 mutations. No mutations in these PAH-predisposing genes were identified in familial PVOD patients.

One variant of BMPR2 was identified in one PVOD patient with bi-allelic mutations in EIF2AK4. This variant was localised in exon 5 (c.604A>T; p.Asn202Tyr) and was not identified in other PAH or PVOD patients, and therefore represents a likely neutral variant.

\section{Consequences of genetic testing in PAH and PVOD patients}

In the presence of a mutation in PAH-predisposing genes

In the presence of a mutation in PAH-predisposing genes in patients (regardless of family history), the patient was classified as having heritable disease (group 1.2 of the classification of pulmonary hypertension) [1, 2]. PAH due to BMPR2, ACVRL1 (ALK1), ENG, KCNK3 or CAV-1 mutations is an autosomal dominant disease with an incomplete penetrance. In BMPR2 mutation carriers, the penetrance is estimated to be $14 \%$ for males and $42 \%$ for females [6]. These patients were informed about the possible risk in their family (parents, siblings and offspring) of developing $\mathrm{PAH}$. We encourage the patient to share this genetic information with their relatives and to inform them about the possibility of genetic counselling and pre-symptomatic diagnosis.

Patients with $\mathrm{PAH}$-causing mutations have a $50 \%$ risk of transmitting the familial mutation to their offspring. In France, the reproductive options for these patients are to remain childless, to adopt, to have a child without genetic testing (reproductive chance), have gamete donation or to undergo pre-implantation diagnosis. Currently, male PAH patients who carry BMPR2 mutations are offered the possibility of pre-implantation diagnosis. Due to the life-threatening risk of pregnancy in $\mathrm{PAH}$, women of child-bearing potential are counselled to avoid pregnancy.

In the absence of mutations detected in a patient displaying sporadic PAH

In this situation, we inform the patient that no predisposing mutation has been identified within the current state of knowledge. Pre-symptomatic genetic testing cannot be offered to other members of the family. Therefore, we recommend no systematic screening for other family members. Patients are informed that it remains possible that a nonidentifiable mutation segregates in the family and the family members are asked to inform us if symptoms suggestive of PAH occur.

\section{In the absence of mutations in a patient displaying a familial form of PAH}

In these circumstances, $\mathrm{PAH}$ patients are informed that an unknown genetic cause probably segregates in the family, but that we are unable to identify it. It is therefore not possible to propose pre-symptomatic diagnosis and pre-implantation diagnosis to family members. All family members should be aware that they are potentially at risk of developing PAH. Careful analysis of genetic trees allows the identification of obligatory carriers of the autosomal dominant genetic trait to whom careful follow-up can be proposed.

\section{Specific issues of genetic counselling in PVOD patients}

PVOD due to EIF2AK4 bi-allelic mutations is an autosomal recessive disease with an undetermined penetrance. Therefore, patients have to be informed about the risk of PVOD for siblings. To date, there are no data on the clinical phenotype of heterozygote carriers of a EIF2AK4 mutation, but we have not observed any signs of PVOD among 29 heterozygote carriers identified in our cohort. The frequency of 
heterozygous in the general population has been estimated to be $<1 / 8000$ [29]. We inform the patients about the legal obligation to share this genetic information with siblings, and to inform them about the possibility of genetic counselling and pre-symptomatic diagnosis.

\section{Genetic counselling in high-risk relatives of PAH or PVOD patients}

Our strategy has allowed us to identify patients with a heritable condition and therefore relatives who are at increased risk for developing PAH or PVOD. When a mutation in a predisposing gene is identified in a patient, genetic counselling and pre-symptomatic genetic diagnosis are available for relatives over 18 years old. Relatives are informed about their risks of carrying the familial mutation, their risk of developing the disease and the transmission of the mutation to their progeny. They also receive complete information about PAH or PVOD symptoms, disease characteristics and prognosis.

All relatives are given the opportunity to have ample time to consider whether to proceed to genetic testing. Moreover, the genetic result is available 1 month after the blood sample. This delay allows relatives the chance to change their mind and suspend their pre-symptomatic diagnosis temporarily or permanently. In France, pre-symptomatic genetic testing for PAH and PVOD is not offered to asymptomatic relatives under 18 years old because it is considered that there are no preventive treatments in PAH or PVOD (table 1).

Up to December 2014, 272 relatives of PAH patients have consented for pre-symptomatic genetic diagnosis (first-degree relatives $n=216$; second-degree relatives $n=56$ ). This has allowed us to identify 99 (36.3\%) asymptomatic carriers of a mutation in PAH-predisposing genes: 96 carriers of a BMPR2 mutation, one carrier of an ACVRL1 (ALK1) mutation and two carriers of a KCNK3 mutation (figure 1).

Regarding autosomal recessive transmission of heritable PVOD, pre-symptomatic diagnosis is only proposed to siblings of PVOD patients carrying bi-allelic EIF2AK4 mutations. Genetic testing was offered to two siblings and none of them carried bi-allelic EIF2AK4 mutations.

We performed a survey in 48 asymptomatic relatives in order to document their reasons for accepting pre-symptomatic genetic diagnosis: $42 \%$ performed genetic testing to inform their own genetic risk, $35 \%$ to inform the genetic risk of their offspring, $10 \%$ for the purpose of childbearing with possible pre-implantation diagnosis, $10 \%$ to inform their family about their genetic status, $8 \%$ in order to know the family branch at risk of $\mathrm{PAH}, 6 \%$ for removing the doubt of being a carrier or not a carrier, $6 \%$ in order to benefit from specialised follow-up if they are carriers of the familial mutation and $6 \%$ for helping research in the field of $\mathrm{PAH}$. One more relative received genetic counselling but declined subsequent genetic testing because of the psychological impact of a positive or negative result. However, 17 relatives (four BMPR2 mutation carriers) who performed genetic testing never returned for genetic consultation to receive their results.

Relatives developing PAH after genetic counselling

Four asymptomatic relatives (one man and three women) developed PAH after genetic counselling with delays of 24, 41, 48 and 96 months. At diagnosis of PAH, three relatives had severe PAH and were in New York Heart Association (NYHA) Functional Class III, with severe hemodynamic impairment (mPAP 34-70 $\mathrm{mmHg}$, cardiac index (CI) $1.66-2.5 \mathrm{~L} \cdot \mathrm{min}^{-1} \cdot \mathrm{m}^{-2}$ ). One relative was diagnosed with mild PAH at 2 months after delivery (NYHA Functional Class II, mPAP $25 \mathrm{mmHg}$, CI $3.77 \mathrm{~L} \cdot \mathrm{min}^{-1} \cdot \mathrm{m}^{-2}$ ). All four patients are currently receiving specific $\mathrm{PAH}$ therapy and are alive.

Consequences of the genetic result in asymptomatic carriers of mutations in PAH-predisposing genes If the familial mutation is not identified in a relative, this person has the same risk of developing PAH as the general population. This person does not require clinical monitoring for PAH and has no risk of transmitting the familial mutation to his/her offspring. In contrast, if the familial BMPR2 mutation is identified in an asymptomatic relative, this person has an estimated risk of $14 \%$ for the development of $\mathrm{PAH}$ if male or $42 \%$ if female. The penetrance of ACVRL1 (ALK1), ENG, KCNK3 and CAV-1 mutations for $\mathrm{PAH}$ is not known. To date, it is not possible to differentiate asymptomatic subjects carrying a mutation who will develop PAH from those who will never develop the disease. Moreover, this person has a $50 \%$ risk of transmitting the disease causing mutation to their offspring. The reproductive options for this person are to remain childless, to adopt, to have a child without genetic testing (reproductive chance), have a gamete donation or to undergo pre-implantation diagnosis.

\section{Follow-up of asymptomatic BMPR2 mutation carriers}

Despite the development of novel therapies, PAH remains a disease with a poor prognosis and for which no cure is possible, apart from lung transplantation in selected cases. However, recent studies have shown that early diagnosis is associated with better long-term outcomes [35]. Thus, it seems important to establish an early diagnosis of $\mathrm{PAH}$, especially in high-risk populations such as asymptomatic carriers of a 
mutation in PAH-predisposing genes. There is no universal consensus or guideline on how to follow this population. However, given the high risk of developing $\mathrm{PAH}$ in these subjects (penetrance ranging from $14 \%$ to $42 \%$ versus 15 to $25 /$ million in the general population), and the limited knowledge of characteristics of this population, it seems essential to offer a prospective follow-up to these individuals. At present, asymptomatic individuals who are tested positive for $\mathrm{PAH}$-causing mutations, and first-degree relatives of heritable PAH patients in whom no causal mutations have been identified, are offered yearly screening echocardiography or when symptoms occur [36]. An ongoing longitudinal study should clarify issues such as optimal screening strategies and predictors of progression to $\mathrm{PAH}$ in asymptomatic BMPR2 carriers (DELPHI-2 study; www.clinicaltrials.gov: NCT01600898).

Lastly, we reported the first pre-implantation diagnosis in a couple with an extensive family history of PAH caused by a BMPR2 mutation [37]. Since then, pre-implantation diagnosis has been offered to two couples and an additional three couples have applied for this procedure. This strategy can be offered to BMPR2 mutation carriers in order to avoid genetic transmission in the offspring. Currently, pre-implantation diagnosis is not proposed when the woman carries the familial mutation, because of the risk of occurrence of the disease in the case of pregnancy and our lack of knowledge of the effect of ovarian stimulation in females with a BMPR2 mutation.

\section{Discussion}

In 12 years of genetic counselling activity, we offered genetic testing to 629 patients displaying PAH or PVOD followed at the French Referral Centre for Pulmonary Hypertension. This systematic research has allowed us to identify 192 patients carrying a mutation in PAH/PVOD-predisposing genes. 26 PVOD patients were found to carry bi-allelic EIF2AK4 mutations. In addition, we identified $12 \mathrm{PAH}$ patients with mutations localised to ACVRL1 (ALK1), two patients with mutations in KCNK3 and 151 patients from 127 different families who carried mutations in BMPR2. These mutations are spread throughout BMPR2 with the exception of exon 13. Mutations were identified mainly in the first part of the protein (extracellular domain and kinase domain) with only $14 \%$ of mutations localised in the cytoplasmic tail domain. It appears that all PAH carriers of an ACVRL1 (ALK1) mutation had either personal signs of HHT and/or that HHT signs could be identified in their family members. This observation suggests that targeted questioning on signs of HHT should be systematically performed in all PAH patients in order to screen for ACVRL1 (ALK1) in the first instance in such a situation. In the absence of personal and familial signs of HHT, screening for ACVRL1 (ALK1) seems unnecessary unless family data are missing. Moreover, it is important to note that during this period, we chose not to screen SMAD9, KCNK3 and CAV-1 and the promoter of $B M P R 2$ in patients considered to have an idiopathic form of $\mathrm{PAH}$ due to the low frequencies of such mutations in familial forms of PAH genes and the difficulties to conclude about the deleterious effect of variants in the promoter region of BMPR2. However, in the future, next-generation sequencing may allow screening all genes involved in vascular disease in all incident PAH/PVOD patients.

It is interesting to note that, although there is only $17 \%$ of mutation carriers in patients displaying apparently idiopathic $\mathrm{PAH}$ compared with $89 \%$ in familial $\mathrm{PAH}$ patients, we have broadly the same absolute numbers of mutation carriers in these two groups: 62 mutation carriers in idiopathic PAH patients and 89 in PAH patients with family history. This observation underscores the importance of proposing genetic counselling and testing to all patients with $\mathrm{PAH}$ considered to be idiopathic in origin. Moreover, as previously reported by THOMSON et al. [38], we identified four patients carrying a de novo mutation in BMPR2. Thus, in the setting of de novo mutation, even if there is no risk of developing the disease for the ascending generation, the offspring have to be informed about their risk and should be followed. Some heritable PAH cases remain without an identifiable genetic mutation. In fact, mutations in PAH-predisposing genes were not identified in $11 \%$ of PAH patients with a family history of disease. These findings suggest that other (as-yet unknown) genes may be involved in the development of PAH and all these remaining families without identified mutations are systematically screened when a suspicion of new PAH-predisposing gene is reported.

Pre-symptomatic genetic diagnosis was proposed to all relatives of mutation carriers and 272 asymptomatic relatives of PAH patients consulted for genetic testing, among whom 99 asymptomatic relatives were found to carry a mutation in PAH-predisposing genes. BMPR2/ACVRL1 (ALK1)/ENG/KCNK3 mutations are characterised by incomplete penetrance for $\mathrm{PAH}$ and to date it is not possible to identify which asymptomatic mutation carriers will ultimately develop PAH. Moreover, neither preventive nor curative treatment of the disease is available and associated genetic or environmental factors contributing to the occurrence of PAH in these mutation carriers are still unknown. Thus, genetic testing in relatives can inform about the carriage status of the familial mutation, but mutation carriers will face other fears and uncertainties because we cannot determine whether they will develop the disease, the age of onset or the progression of the disease. Thus, the results of genetic testing among asymptomatic mutation carriers may 
have a significant psychological impact, with feelings of fear and anxiety. Some relatives have reported feelings of guilt for transmitting the mutation to their children or not to have developed the disease unlike other family members. The study of our cohort revealed that the results of genetic testing also have an impact on relatives who do not carry the familial mutation. Although they are reassured about not having the risk of developing PAH and not having the risk of transmitting the mutation to their offspring, some display feelings of guilt, anger, cowardice, injustice and abandonment. For all these reasons, appropriate psychological support is mandatory throughout the pre-symptomatic diagnosis process, and careful counselling must be provided regarding the advantages and disadvantages of knowing their genetic status.

It has been shown that early diagnosis and timely intervention in PAH may translate into better long-term outcomes [35, 39]. Pre-symptomatic genetic screening can be of interest because it will result in careful and regular clinical follow-up of asymptomatic mutation carriers and facilitate early PAH diagnosis. Therefore, at the French Referral Centre for Pulmonary Hypertension, we offer all asymptomatic BMPR2 mutation carriers a programme to detect the disease at an early phase. This clinical screening includes a clinical examination, an ECG and a yearly echocardiogram or whenever disease symptoms occur. However, no strategy has been validated and we have initiated a research programme since March 2012 (DELPHI-2 study; www.clinicaltrials.gov: NCT01600898) in order to define the optimal screening strategy and the frequency of screening in these asymptomatic mutation carriers.

Finally, the identification of a BMPR2 mutation in asymptomatic subjects or in male PAH patients may also lead to the consideration of pre-natal diagnosis or pre-implantation diagnosis. These two techniques are used to avoid the birth of an individual with a mutation that can result in a life-threatening and incurable disease. These techniques are used in other diseases such as Huntington's disease or cystic fibrosis, but they are still subject to debate in PAH because of the incomplete penetrance of BMPR2 mutations. Due to the severity of this devastating disease and the potential impact of abortion on parents, our group is in favour of the technique of pre-implantation diagnosis in selected couples after collegial and ethical discussion.

In conclusion, BMPR2, ACVRL1 (ALK1), ENG, KCNK3 and CAV-1 mutations predispose to PAH development, and bi-allelic EIF2AK4 mutations predispose to PVOD. Our 12-year experience demonstrates that genetic counselling can be implemented in referral centres for pulmonary hypertension. More data are needed to validate a PAH screening algorithm in asymptomatic BMPR2 mutation carriers.

\section{References}

1 Humbert M, Sitbon O, Simonneau G. Treatment of pulmonary arterial hypertension. N Engl J Med 2004; 351: $1425-1436$.

2 Galiè N, Humbert M, Vachiery J-L, et al. 2015 ESC/ERS Guidelines for the diagnosis and treatment of pulmonary hypertension. Eur Heart J 2015; in press [DOI: 10.1093/eurheartj/ehv317].

3 Galiè N, Humbert M, Vachiery J-L, et al. 2015 ESC/ERS Guidelines for the diagnosis and treatment of pulmonary hypertension. Eur Respir J 2015; 46: 903-975.

4 Simonneau G, Robbins IM, Beghetti M, et al. Updated clinical classification of pulmonary hypertension. J Am Coll Cardiol 2009; 54: S43-S54.

5 Dresdale DT, Michtom RJ, Schultz M. Recent studies in primary pulmonary hypertension, including pharmacodynamic observations on pulmonary vascular resistance. Bull N Y Acad Med 1954; 30: 195-207.

6 Larkin EK, Newman JH, Austin ED, et al. Longitudinal analysis casts doubt on the presence of genetic anticipation in heritable pulmonary arterial hypertension. Am J Respir Crit Care Med 2012; 186: 892-896.

7 Nichols WC, Koller DL, Slovis B, et al. Localization of the gene for familial primary pulmonary hypertension to chromosome 2q31-32. Nat Genet 1997; 15: 277-280.

8 Morse JH, Jones AC, Barst RJ, et al. Mapping of familial primary pulmonary hypertension locus (PPH1) to chromosome 2q31-q32. Circulation 1997; 95: 2603-2606.

9 Lane KB, Machado RD, Pauciulo MW, et al. Heterozygous germline mutations in BMPR2, encoding a TGF-beta receptor, cause familial primary pulmonary hypertension. Nat Genet 2000; 26: 81-84.

10 Deng Z, Morse JH, Slager SL, et al. Familial primary pulmonary hypertension (gene PPH1) is caused by mutations in the bone morphogenetic protein receptor-II gene. Am J Hum Genet 2000; 67: 737-744.

11 Miyazono K, Maeda S, Imamura T. BMP receptor signaling: transcriptional targets, regulation of signals, and signaling cross-talk. Cytokine Growth Factor Rev 2005; 16: 251-263.

12 Fujii M, Takeda K, Imamura T, et al. Roles of bone morphogenetic protein type I receptors and Smad proteins in osteoblast and chondroblast differentiation. Mol Biol Cell 1999; 10: 3801-3813.

13 Tuder RM, Archer SL, Dorfmüller P, et al. Relevant issues in the pathology and pathobiology of pulmonary hypertension. J Am Coll Cardiol 2013; 62: D4-D12.

14 Machado RD, Eickelberg O, Elliott CG, et al. Genetics and genomics of pulmonary arterial hypertension. J Am Coll Cardiol 2009; 54: S32-S42.

15 Rosenzweig EB, Morse JH, Knowles JA, et al. Clinical implications of determining BMPR2 mutation status in a large cohort of children and adults with pulmonary arterial hypertension. J Heart Lung Transplant 2008; 27: 668-674.

16 Girerd B, Montani D, Coulet F, et al. Clinical outcomes of pulmonary arterial hypertension in patients carrying an ACVRL1 (ALK1) mutation. Am J Respir Crit Care Med 2010; 181: 851-861.

17 Aldred MA, Machado RD, James V, et al. Characterization of the BMPR2 5'-untranslated region and a novel mutation in pulmonary hypertension. Am J Respir Crit Care Med 2007; 176: 819-824. 
Cogan JD, Pauciulo MW, Batchman AP, et al. High frequency of BMPR2 exonic deletions/duplications in familial pulmonary arterial hypertension. Am J Respir Crit Care Med 2006; 174: 590-598.

19 Machado RD, Southgate L, Eichstaedt CA, et al. Pulmonary arterial hypertension: a current perspective on established and emerging molecular genetic defects. Hum Mutat 2015; 36: 1113-1127.

20 Thomson J, Machado R, Pauciulo M, et al. Familial and sporadic primary pulmonary hypertension is caused by BMPR2 gene mutations resulting in haploinsufficiency of the bone morphogenetic protein type II receptor. J Heart Lung Transplant 2001; 20: 149.

21 Chaouat A, Coulet F, Favre C, et al. Endoglin germline mutation in a patient with hereditary haemorrhagic telangiectasia and dexfenfluramine associated pulmonary arterial hypertension. Thorax 2004; 59: 446-448.

22 Nasim MT, Ogo T, Ahmed M, et al. Molecular genetic characterization of SMAD signaling molecules in pulmonary arterial hypertension. Hum Mutat 2011; 32: 1385-1389.

23 Shintani M, Yagi H, Nakayama T, et al. A new nonsense mutation of SMAD8 associated with pulmonary arterial hypertension. J Med Genet 2009; 46: 331-337.

24 Austin ED, Ma L, LeDuc C, et al. Whole exome sequencing to identify a novel gene (Caveolin-1) associated with human pulmonary arterial hypertension. Circ Cardiovasc Genet 2012; 5: 336-343.

25 Ma L, Roman-Campos D, Austin ED, et al. A novel channelopathy in pulmonary arterial hypertension. $N$ Engl $J$ Med 2013; 369: 351-361.

26 Mandel J, Mark EJ, Hales CA. Pulmonary veno-occlusive disease. Am J Respir Crit Care Med 2000; 162: 1964-1973.

27 Montani D, Price LC, Dorfmuller P, et al. Pulmonary veno-occlusive disease. Eur Respir J 2009; 33: 189-200.

28 Montani D, Achouh L, Dorfmüller P, et al. Pulmonary veno-occlusive disease: clinical, functional, radiologic, and hemodynamic characteristics and outcome of 24 cases confirmed by histology. Medicine (Baltimore) 2008; 87: 220-233.

29 Eyries M, Montani D, Girerd B, et al. EIF2AK4 mutations cause pulmonary veno-occlusive disease, a recessive form of pulmonary hypertension. Nat Genet 2014; 46: 65-69.

30 Sitbon $\mathrm{O}$, Humbert $\mathrm{M}$, Nunes $\mathrm{H}$, et al. Long-term intravenous epoprostenol infusion in primary pulmonary hypertension: prognostic factors and survival. J Am Coll Cardiol 2002; 40: 780-788.

31 Sitbon O, Humbert M, Jaïs X, et al. Long-term response to calcium channel blockers in idiopathic pulmonary arterial hypertension. Circulation 2005; 111: 3105-3111.

32 Laveneziana P, Montani D, Dorfmüller P, et al. Mechanisms of exertional dyspnoea in pulmonary veno-occlusive disease with EIF2AK4 mutations. Eur Respir J 2014; 44: 1069-1072.

33 Girerd B, Coulet F, Jaïs X, et al. Characteristics of pulmonary arterial hypertension in affected carriers of a mutation located in the cytoplasmic tail of bone morphogenetic protein receptor type 2. Chest 2015; 147: 1385-1394.

34 Sztrymf B, Coulet F, Girerd B, et al. Clinical outcomes of pulmonary arterial hypertension in carriers of BMPR2 mutation. Am J Respir Crit Care Med 2008; 177: 1377-1383.

35 Galiè N, Rubin L, Hoeper M, et al. Treatment of patients with mildly symptomatic pulmonary arterial hypertension with bosentan (EARLY study): a double-blind, randomised controlled trial. Lancet 2008; 371: 2093-2100.

36 Soubrier F, Chung WK, Machado R, et al. Genetics and genomics of pulmonary arterial hypertension. J Am Coll Cardiol 2013; 62: D13-D21.

37 Frydman N, Steffann J, Girerd B, et al. Pre-implantation genetic diagnosis in pulmonary arterial hypertension due to BMPR2 mutation. Eur Respir J 2012; 39: 1534-1535.

38 Thomson JR, Machado RD, Pauciulo MW, et al. Sporadic primary pulmonary hypertension is associated with germline mutations of the gene encoding BMPR-II, a receptor member of the TGF-beta family. J Med Genet 2000; 37: 741-745.

39 Humbert M, Yaici A, de Groote P, et al. Screening for pulmonary arterial hypertension in patients with systemic sclerosis: clinical characteristics at diagnosis and long-term survival. Arthritis Rheum 2011; 63: 3522-3530. 